\title{
Effectiveness of Oil-layered Albumin Microbubbles Produced using Microfluidic T-junctions in Series for In-vitro Inhibition of Tumor Cells
}

\author{
Aaqib H. Khan ${ }^{\#}$, Xinyue Jiang ${ }^{\dagger}$, Swarupkumar Surwase ${ }^{\#}$, Merve Gultekinoglu ${ }^{\ddagger}$, Cem Bayram ${ }^{\S}$ Indumathi Sathisaran ${ }^{\&}$, \\ Dhiraj Bhatia $^{\&}$, Jubair Ahmed ${ }^{\dagger}$, Bingjie Wu ${ }^{\dagger}$, Kezban Ulubayram ${ }^{\ddagger}$, Mohan Edirisinghe ${ }^{\dagger}$, and Sameer V. Dalvi*** \\ \# Chemical Engineering, Indian Institute of Technology Gandhinagar, Palaj, Gandhinagar - 382355, Gujarat, India \\ †Department of Mechanical Engineering, University College London (UCL), London WC1E 7JE, U.K. \\ \$Department of Basic Pharmaceutical Sciences, Faculty of Pharmacy, Bioengineering Division, Institute for \\ Graduate Studies in Science \& Engineering, Hacettepe University, Ankara 06100, Turkey

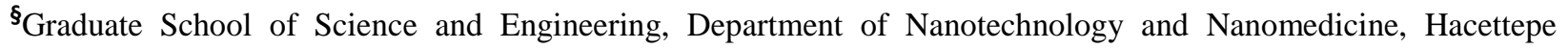 \\ University, Ankara 06800, Turkey \\ \& Biological Engineering, Indian Institute of Technology Gandhinagar, Palaj, Gandhinagar - 382355, Gujarat, India
}

\begin{abstract}
This work focuses on the synthesis of oil-layered microbubbles using two microfluidic T-junctions in series and evaluation of the effectiveness of these microbubbles loaded with doxorubicin and curcumin for cell invasion arrest from 3D spheroid models of triple negative breast cancer (TNBC), MDA-MB-231 cell line. Albumin microbubbles coated in drugladen oil layer were synthesized using a new method of connecting two microfluidic T-mixers in series. Double-layered microbubbles thus produced consist of an innermost core of nitrogen gas encapsulated in an aqueous layer of bovine serum albumin (BSA) which in turn, is coated with an outer layer of silicone oil. In order to identify the process conditions leading to the formation of double-layered microbubbles, a regime map was constructed based on Capillary numbers for aqueous and oil phases. The microbubble formation regime transitions from double-layered to single layer microbubbles and then to formation of single oil droplets upon gradual change in flow rates of aqueous and oil phases. In-vitro dissolution studies of double-layered microbubbles in an air-saturated environment indicated that a complete dissolution of such bubbles produces
\end{abstract}


an oil droplet devoid of gas bubble. Incorporation of doxorubicin and curcumin was found to produce a synergistic effect, which resulted in higher cell deaths in 2D monolayers of TNBC cells and inhibition of cell proliferation from 3D spheroid models of TNBC cells compared to control.

Keywords: Double layered microbubbles, albumin microbubbles, 3D spheroids, curcumin, doxorubicin, dissolution

*E-mail: sameervd@iitgn.ac.in; Phone: 091-7923952408 
Introduction: Microbubbles are increasingly being used in acoustic imaging ${ }^{1}$, targeted drug $^{2-4}$ and gene delivery ${ }^{5,6}$. In presence of ultrasound, microbubbles generate acoustic backscatter, which can be used to image organs ${ }^{7}$. However, the efficacy of microbubbles as ultrasound contrast agents decreases with an increase in the polydispersity of the microbubble population ${ }^{8}$. There are numerous techniques available to produce microbubbles for example, probe sonication $^{9,}{ }^{10}$, amalgamation ${ }^{11}, 12$, freeze-drying ${ }^{13}$, microfluidic devices ${ }^{14-18}$, etc. Out of all these techniques, the monodispersity of microbubbles produced using microfluidic devices is unparalleled to any other microbubble synthesis technique. Several designs of microfluidic devices exist in the literature. Most common devices reported are T-junction ${ }^{19-22}$, v-junction and co-flow $^{23,24}$, and flow-focusing microfluidic devices (FFMD) ${ }^{25,}$ 26. T-Junction microfluidic device is a cross-flow type microbubble formation device where the microbubbles are formed due to the instability of the gas-liquid interface. The instability is mainly caused by shear stress and pressure forces acting on the gas-liquid interface. The outlet of the T-junction contains a sequence of the gas and liquid phases such that the gas pocket is contained within two consecutive liquid droplets ${ }^{27}, 28$. At the tip of the $\mathrm{T}$ - junction, the liquid stream emerges out first and encapsulates the incoming gas pocket.

Unlike single-layer microbubbles where the amount of drug loading is restricted by the size of the microbubble and the encapsulating material, the drug loading capacity can be enhanced by adding an oil-layer on the microbubbles. Researchers have used microfluidic devices to form such multilayered microbubbles as well ${ }^{29-33}$. These multilayered bubbles mainly consisted of gas core encapsulated in an oil or aqueous layer which in turn was coated in an aqueous or oil layer respectively. Wan and Stone ${ }^{33}$ reported the encapsulation of a high volume fraction of gas bubble/multiple gas bubbles in a water-oil system. Monodisperse microbubbles were formed by 
the flow focusing technique by contacting the gas phase and water phase following which the bubbles surrounded by water were dispersed in the continuous oil phase. Arakawa et al. ${ }^{29}$ report a method to produce organic phase coated gas core microbubbles dispersed in a water phase. Bubbles were formed at the lumped junction of the three channels, the gas was encapsulated by the organic phase and dispersed in the main channel containing water. The bubble generation rate was 40 bubbles/ sec, and the diameter of bubbles varied between $110-220 \mu \mathrm{m}$. Both these reports, however, did not provide insight to the dynamics of the formation multilayered microbubbles. Shih et al. ${ }^{32}$ used a flow-focusing technique to produce monodisperse drug loadable microbubbles. Lipid was used as an encapsulating layer and oil as the drug carrier. The authors show that the microbubble formation process transitions from geometry controlled to dripping regime upon increasing the lipid solution flow rate. The authors report that transition in the microbubbles formation regime is a competition between viscous and surface tension forces. The authors report that the hydrophobic affinity of the oil and gas phase caused the oil to "act as a glue" between aqueous lipid and gas phase to produce double layered microbubbles.

Wang et al. ${ }^{34}$ used a co-axial microfluidic setup to produce gas in oil in water double emulsions. Bubbles were formed at the exit of the co-axial capillary by the contact of gas with oil and water stream. The formation of microbubbles in the microfluidic setup, growth of the gas bubble and break up, was dependent upon the flow rate of the outer water stream. Sang et al. ${ }^{31}$ later used a dual co-axial microfluidic device for the formation of Gas/Liquid/Liquid hollow droplets. The authors modeled microbubble formation in oil-water system using scaling laws. The formation of hollow droplets was described as a two stage process; the filling or expansion stage and the necking stage. The filling stage is described as the stage when the fluid occupies a critical volume in the outlet capillary such that the drag force acting on the fluid is higher than the 
interfacial surface tension force. The necking stage is described as the axial stretching and radial shrinkage of the fluid streams due to the drag force exerted by the liquid stream. The authors compared the filling and the necking stages of the gas-oil-water system with the gas-liquid and liquid-liquid systems. They show that the filing stage is similar to the filing stage in the gasliquid system and the necking stage resembles the liquid-liquid system. Recently, Churchman et al. ${ }^{35}$ employed a flow-focusing technique to form oil and lipid encapsulated microbubbles. The authors utilize a new approach that involves the formation of microbubbles by self-assembly of lipid-coated oil nanodroplets at the gas-liquid interface. The authors proposed a two-step mechanism for microbubble formation: (i) adsorption of lipid nanodroplets on the bubble surface and formation of a heterogeneous, non-uniform layer of oil droplets coated with lipid molecules, and (ii) spreading of nanodroplets over the bubble surface followed by drainage of excess oil from the interface.

In this work, we present a new method for the production of double-layered microbubbles with the oil as an outer layer, the aqueous protein solution as the middle layer, and nitrogen (N2) gas as the core of microbubbles. Double-layered microbubbles are formed using two microfluidic Tjunctions in series by sequential coating of the oil phase on the aqueous phase which encapsulates the gas slug. Further, a regime map for the formation of double-layered microbubbles was constructed by plotting the capillary number for aqueous phase against the capillary number for oil phase. The double-layered microbubbles were loaded with curcumin and doxorubicin and were tested on 2D monolayer and 3D spheroid models of triple-negative breast cancer cells, MDA-MB-231. 


\section{Experimental}

Materials: Bovine serum albumin (BSA) (98\% Lyophilized powder) was purchased from Proliant Biologicals, New Zealand; sodium dodecyl sulphate (SDS) was purchased from SigmaAldrich (India) and used without further purification. Nitrogen (Ultrahigh pure grade) used for microbubble preparation was purchased from JP gas suppliers, India. Phosphate buffered saline (PBS) was purchased from Sigma-Aldrich, India. Bovine serum albumin (BSA) was used as a model protein for the synthesis of microbubbles. Linear Silicone oil (70 cP) was purchased from Kusum oils, Mumbai, India. 3D tumor models of MDA-MB-231 (M. D. Anderson - Metastasis Breast- 231) cells were generated using the established protocol ${ }^{36}$. The MDA-MB-231 cells were cultured and grown in an Esco incubator in $5 \% \mathrm{CO}_{2}$ and $85 \%$ Relative Humidity (RH) condition at $37^{\circ} \mathrm{C}$. Phalloidin-Alexa Fluor488 stain was procured from Invitrogen. The TissueCulture (TC) treated T25 flasks used for growing MDA-MB-231 cells were obtained from Cole Parmar Ltd. Dulbecco's Modified Eagle's Medium (DMEM) - high glucose, PenicillinStreptomycin Solution (Pen-Strep Sol), Trypsin-EDTA solution, Gibco Fetal Bovine Serum Albumin (FBS), Phosphate Buffered Saline (PBS) (pH 7.2), 4\% paraformaldehyde (4\% PFA) and Triton X-100 was purchased from Sigma Aldrich Pvt. Ltd. Collagen I - Rat tail from Thermo Fisher Scientific was utilized as Extra-Cellular Matrix (ECM) for conducting cell invasion assays. Hoechst 33258 solution and Mowiol 4-88 mounting medium were bought from Merck Ltd. 96-well plates and 12-well plates used for cytotoxicity and cellular uptake assays were purchased from Corning Pvt. Ltd.

Preparation of solutions for microbubble production: Aqueous BSA solution $(15 \% \mathrm{w} / \mathrm{w})$, was prepared by dissolving BSA in deionized water at room temperature with constant magnetic stirring for $1 \mathrm{hr}$. SDS at below CMC concentration of $3.5 \mathrm{mM}$ was added to the BSA solution to 
decrease the surface tension of the gas-liquid interface. SDS was added at below CMC levels to avoid micelles formation. Due to the higher viscosity of glycerol, it was added to the aqueous solution of SDS and BSA in proportion of $15 \%(\mathrm{v} / \mathrm{v})$ to increase the viscosity of the solution. Silicone oil of viscosity $70 \mathrm{mPa}$.s was used as an oil phase $\mathrm{e}^{37,38}$.

Microfluidic Apparatus: Microfluidic T-junction was custom made using a $25 \mathrm{~mm}$ x 25mm x $15 \mathrm{~mm}$ PDMS block. The PDMS block consisted of three openings, one for gas inlet and the other for liquid inlet and the other for microbubble outlet respectively. Teflon FEP tubings with internal diameter of $200 \mu \mathrm{m}$ were embedded into the PDMS block (Fig. 1A). The tubings were connected to the PDMS block using high performance fluidic connectors to secure the system from leakage. The tubings were arranged to allow cross flow mixing of liquid and gas phases. The mixing zone for the gas and liquid phases was kept similar to $200 \mu \mathrm{m}$ which is similar to the inner diameter of the tubes. Two $\mathrm{T}-$ junctions were assembled in series such that the bubbles formed in the first $\mathrm{T}-$ junction, were encapsulated by the liquid phase in the second $\mathrm{T}-$ junction to form double-layered microbubbles. The upper tubing of the $\mathrm{T}-\mathrm{Junctions}$ was connected to a gas supply unit and the horizontal tubing (Fig. 1A) for liquid inlet was connected to syringe pumps to ensure constant non-pulsating liquid flow into the channels.

Optical microscopy imaging and characterization of microbubbles: Microbubble formation in the $\mathrm{T}$ - junction was captured using a Photron FASTCAM mini UX high speed camera (Fig 1 B\&C). The videos were recorded at 4000 frames/sec. In order to distinguish between different immiscible liquid streams, methylene blue was added to the BSA stream (aqueous phase). Microbubble formation process in both the T- junctions was recorded sequentially at a constant liquid flow rate and gas pressure for every experiment. 

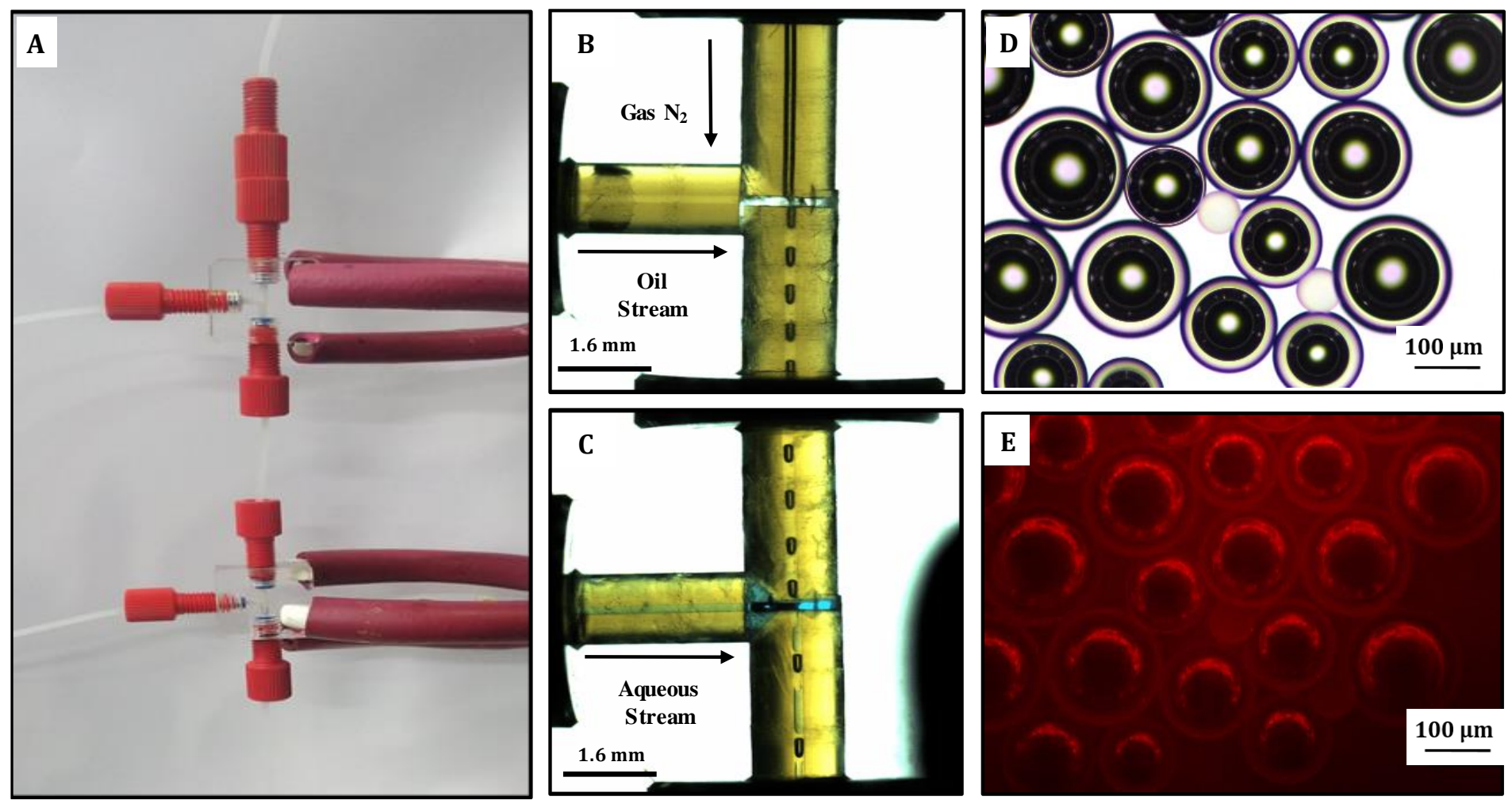

Figure 1: (A) Experimental setup of T - junctions connected in series (B) High speed camera image of 1st T- junction (C) High speed camera image of 2 nd $\mathbf{T}$ - junction. Methylene blue was added to aqueous phase in the 2 nd $\mathbf{T}$-junction to distinguish between oil and aqueous stream (D) Optical image of double-layered microbubbles and (E) Fluorescent image of doublelayered microbubbles. Rhodamine B was added to aqueous phase to identify the presence of oil and aqueous phase in the double layered microbubble 
Initially, a stable flow condition was identified to produce double layered microbubbles. For a given oil flow rate, the aqueous phase flow rate and gas pressure was adjusted such that the system produces double layered microbubbles. Once the stable condition of $0.045 \mathrm{ml} / \mathrm{min}$ of oil phase flow, $0.075 \mathrm{ml} / \mathrm{min}$ of aqueous phase and $101.3 \mathrm{kN} / \mathrm{m}^{2}$ of gas pressure (gauge pressure) was achieved, the oil flow rate and the gas pressure was held constant and the aqueous phase flow rate was varied until the destabilization of the flow occurred leading to microbubble formation. Double layered microbubbles were collected over a glass slide and collection vials containing DI water as the collection phase and were imaged immediately under microscope with 10X objective lens (Nikon Eclipse Ni upright microscope). Optical images captured using the microscope were exported to an image processing software, Image J, to measure the diameter of the microbubble. Fluorescence imaging was performed to confirm the location of aqueous layer and oil layer on microbubbles. Aqueous phase was stained with Rhodamine B. Fluorescence images were captured under a Nikon Eclipse Ni upright microscope with mercury lamp (Nikon Model C-SHG1).

Estimation of microbubble production rate in two $\mathbf{T}$ - mixers: High speed videos captured during microbubble formation were used to find out the production rate of the microbubble formation. Frame rates to capture the microbubble formation process was 4000 frames/sec. Production rate (bubble/sec) was calculated using Equation 1. Number of microbubbles pinched off in a recorded video was calculated as the bubble count.

$$
\text { Bubble production rate }\left(\frac{\text { Bubbles }}{\mathrm{Sec}}\right)=\frac{\text { Bubble count } \times \frac{\text { Frames }}{\mathrm{Sec}}}{\text { Total no. of frames }}
$$

In-vitro stability of Double Layered Microbubbles: In-vitro dissolution behavior of double layered microbubbles was observed in a transparent dissolution chamber made of acrylic. The sample volume of the chamber was $\sim 2 \mathrm{~mL}$. The chamber was sealed and made airtight after 
addition of microbubbles to ensure that the dissolution is not affected due to an exposure to an ambient air. Freshly prepared double layered microbubble suspensions were injected into the dissolution chamber. The microbubble dissolution was observed using a Nikon Eclipse Ni upright microscope with 10X or 20X objective lens. The time-lapse videos were recorded using a high-resolution DS fi2 camera of the microscope. A single double layered microbubble in the dissolution chamber was filmed until its complete dissolution. The snapshots were taken from the recorded video and processed using Image J Software to obtain the Radius v/s Time plots. All the experiments were performed at a room temperature of $\sim 25^{\circ} \mathrm{C}$.

In-vitro Drug release from double layered microbubbles: The kinetics of drug release from double-layered microbubbles, in presence and absence of ultrasound, was estimated using UV-vis spectrophotometry. Microbubbles were synthesized using two T-junctions in series. CUR was dissolved in ethanol at a concentration of $27 \mathrm{mM}$ in order to prepare a stock solution. The stock solution was centrifuged at $14462 \mathrm{~g}$ for $5 \mathrm{mins}$ and $200 \mu \mathrm{l}$ of the supernatant was added to the aqueous phase. $2 \mathrm{mg}$ of CUR dispersed in $10 \mathrm{~mL}$ Silicone oil was used as oil phase. Approximately $5 \times 10^{3}$ freshly prepared curcumin loaded double-layered microbubbles were collected in a vial and added to $5 \mathrm{~mL}$ of $20 \%$ Ethanol and water solution. The temperature of the solution was maintained at $37{ }^{\circ} \mathrm{C}$. To estimate the effect of ultrasound on drug release from the microbubbles, one of the test samples was sonicated at an intensity of $0.5 \mathrm{~W} / \mathrm{cm}^{2}$ for $30 \mathrm{~s}$, using Electroson 608 ultrasonic system with $1 \mathrm{MHz}$ mean frequency in 1:1 pulse mode (2 ms ON and $2 \mathrm{~ms}$ OFF cycle) ${ }^{9}$. The drug release study was conducted for 24 hours and repeated three times. At regular intervals, $100 \mu \mathrm{L}$ of the sample was pipetted out from the above solution and centrifuged at $14462 \mathrm{~g}$. The supernatant was analyzed using UV-vis spectroscopy. The absorbance value was inserted to the calibration curve for $20 \%$ Ethanol water solution to obtain 
the concentration of curcumin released at each interval. The extent of drug release was found out by Equation 2.

Drug released $=\{$ Concentration of drug in sample $*[$ Initial media volume $-[$ Sample volume $*(n-$ $1)]]\}+\Sigma_{\mathrm{n}-1}$ [Concentration of drug in the sample* Sample volume]

In-vitro cytotoxicity and LIVE/DEAD assays: Cytotoxicity evaluation of double layered microbubbles loaded with varying concentrations of doxorubicin and curcumin were carried out with MDA-MB-231 breast cancer cells in 2D culture conditions. The cells were seeded at a concentration of $2 \times 10^{4}$ cells $/ \mathrm{mL}$ into a 48 well plate $37^{\circ} \mathrm{C}$ in a $90 \%$ humidified incubator with $5 \% \mathrm{CO}_{2}$, and incubated overnight with growth media consisting Dulbecco's Modified Eagle's Medium (DMEM) supplemented 10\% Fetal Bovine Serum (FBS). The day after the overnight incubation, microbubbles prepared with varying active agent concentrations were added into cells at a density of $10^{3}$ bubbles per cell and the samples were agitated for $10 \mathrm{~s}$ with ultrasonic bath and left to incubate for 48 h. $50 \mu \mathrm{L}$ of MTT (3-(4,5-Dimethylthiazol-2-Y1)-2,5Diphenyltetrazolium Bromide) agent at a concentration of $1 \mathrm{mg} / \mathrm{mL}$ was added per well and after 4 hours all supernatants were removed and $200 \mu \mathrm{L}$ dimethyl sulfoxide (DMSO) was added to dissolve purple formazan crystals. Relative cell viabilities against negative control group were calculated by the absorbance spectra at $570 \mathrm{~nm}$ wavelength. The cell viabilities were also investigated with fluorescence based LIVE/DEAD assay kit (Invitrogen, Paisley, UK). After 48 $\mathrm{h}$ incubation with microbubbles, cell media and microbubble residue were removed from the wells and subsequently PBS solution containing $2 \mu \mathrm{M}$ calcein $\mathrm{AM}$ and $4 \mu \mathrm{M}$ ethidium homodimer-1 was added. Cells interacted with fluorescence dyes at dark for 45 min and representative images of green (live) and red (dead) cells were shot with fluorescence microscopy (Leica Microsystems, Germany). 
Cell Invasion Studies: Cell invasion assay for double layered microbubbles loaded with curcumin (CUR) was carried out on 3D spheroid models of a Triple Negative Breast Cancer (TNBC) cell line, MDA-MB-231. Collagen I - Rat Tail obtained from Thermo Fisher Scientific was used as the Extra-Cellular Matrix (ECM) in this experiment. CUR was dissolved in ethanol at a concentration of $27 \mathrm{mM}$ in order to prepare a stock solution. The stock solution was centrifuged at $14462 \mathrm{~g}$ for $5 \mathrm{mins}$ and $200 \mu \mathrm{l}$ of the supernatant was added to $15 \mathrm{wt} \%$ BSA solution. The resultant final CUR-BSA solution was used as the aqueous phase for double layered microbubble synthesis. $2 \mathrm{mg}$ of CUR dispersed in $10 \mathrm{~mL}$ Silicone oil was used as oil phase. MDA-MB-231 3D spheroids used for the cell invasion studies were prepared by hanging drop culture method ${ }^{36}$. For preparation of hanging drops, a T25 flask with $85 \%$ confluency was trypsinized using $1 \mathrm{~mL}$ trypsin. $4 \mathrm{~mL}$ of fresh complete medium was added to the trypsinized solution and mixed well. The medium containing cells were centrifuged at $734 \mathrm{~g}$ for a period of 10 minutes. The obtained pellet was resuspended in $4 \mathrm{~mL}$ fresh complete medium. The $35 \mu \mathrm{L}$ of cells contained in the medium were added in the form of drops over the inner surface of the petri dish lid. Around $30 \mathrm{~mL}$ Phosphate Buffer Saline (PBS) was added to the Petri dish base for providing moisture environment for spheroid growth. The Petri dish lid containing cell droplets was placed upside down covering the Petri dish base. The cells were allowed to grow until spheroid formation was observed. The well-formed spheroids were harvested from the hanging drop at $36^{\text {th }}$ hour. These harvested spheroids were then used for cell invasion assays. One hanging drop comprises one tumor spheroid. The tumor spheroid contained in the hanging drop was harvested and dispersed in $50 \mu \mathrm{L}$ of ECM-complete medium (at 2:1 volume ratio) matrix in a 12-well plate. The spheroids were then treated with appropriate samples. The appropriate controls and the spheroids subjected to different treatments were then incubated in incubator 
containing $5 \% \mathrm{CO}_{2}$ at $98 \%$ Relative Humidity (RT) and $37{ }^{\circ} \mathrm{C}$. The tumor models investigated in this assay comprises the $3 \mathrm{D}$ spheroid at $0^{\text {th }}$ hour, 3D spheroid without any treatment (negative control) after 24 hours, 3D spheroid treated with raw CUR (as a positive control) after 24 hours, 3D spheroids treated with $20 \mu \mathrm{L}$ of CUR loaded microbubbles after 24 hours in presence and absence of ultrasound treatment at an intensity of $0.5 \mathrm{~W} / \mathrm{cm}^{2}$ for $10 \mathrm{sec}$ (The concentration of microbubbles used was maintained as $\sim 10^{3}-10^{4} \# / \mathrm{mL}$ in all the samples). This condition mimics the physiological condition of the tumor clumps in the in vivo environment. After 24 hours, the spheroids with ECM were carefully isolated and fixed using $4 \%$ paraformaldehyde (4 \% PFA) solution followed by staining with Hoechst and Phalloidin-Alexa 488 stains. After staining, the spheroids were mounted using mowiol as mounting medium on the glass slide for confocal microscopic image analysis using Leica DMI8 Confocal microscope. The excitation wavelengths $405 \mathrm{~nm}$ and $488 \mathrm{~nm}$ were used for confocal imaging of the 3D tumor models. The migration distances of tumor cells from the 3D tumor models were calculated using ImageJ software. The Cell Invasion Indices of the tumor models were further calculated using the following equation ${ }^{39}$, Equation 3:

$$
\text { Cell invasion index }=\frac{(\text { Distance migrated by cells from surface of the spheroid })}{(\text { Diameter of the core spheroid })}
$$

It should be noted that the distance migrated by the tumor cells from the 3D spheroid model at $0^{\text {th }}$ hour is the diameter of the spheroid harvested as fresh from the hanging drop culture.

\section{Results and Discussion}

Production and characterization of double-layered microbubbles: Fig. 1 presents the pictures of a double T-junction device used to produce double layered microbubbles. Figs. 1D and $1 \mathrm{E}$ present the optical and fluorescent microscopic images of double layered microbubbles. Rhodamine B was used to stain the aqueous BSA solution. Microbubbles produced using the 
double T-junction device were imaged under florescence microscope to identify presence and sequence of liquid BSA solution and silicon oil layers on the double layered microbubble. It could be observed from the fluorescent images (Fig. 1E) that the inner layer of the doublelayered microbubbles was fluorescent while the outer layer was not, indicating that the inner layer of microbubbles was the aqueous albumin layer and the outer layer was the oil layer.

Fig. 2A presents the average size of double layered microbubbles as a function of the ratio of aqueous phase flow rate $\left(\mathrm{Q}_{\mathrm{Aq}}\right)$ to oil phase flow rate $\left(\mathrm{Q}_{\text {oil }}\right)$ (as black dotted line). Unlike single $\mathrm{T}$ junction setup where the reduction in microbubble size is dependent upon the fluid properties and the cross flow shear offered by the single liquid stream ${ }^{20}$, in case of two T-junction setup, the size of the bubble is affected by the liquid streams in both the T-junctions. Fig. 5 shows that the microbubble diameter decreased linearly as flow ratio $\left(\mathrm{Q}_{\mathrm{Aq}} / \mathrm{Q}_{\mathrm{Oil}}\right)$ increased from 0 to 5 , and then became steady with an increase in flow ratio beyond 5. A linear decrease in the microbubble size at lower flow ratios (0-5) suggests that with an increase in $\mathrm{Q}_{\mathrm{Aq}} / \mathrm{Q}_{\text {oil }}$ caused by an increase in aqueous flow rate $\left(\mathrm{Q}_{\mathrm{Aq}}\right)$ in the second $\mathrm{T}$-junction, the net downward force exerted by the gas stream (in the first $\mathrm{T}$-junction) at a constant gas pressure decreases which then reduces bubble size in the second T-junction due to increased splitting of the incoming gas slug as a result of the high cross-flow shear applied by the aqueous stream. Similar observation was reported by Jiang et al. ${ }^{22}$ during experimental investigation to produce single microbubbles using two T-junctions where the authors showed that the diameter of the bubble is dependent on the combined flow rate of fluids in two T-junctions and observed a linear decrease in diameter with respect to flow rates of gas and liquid phases. 

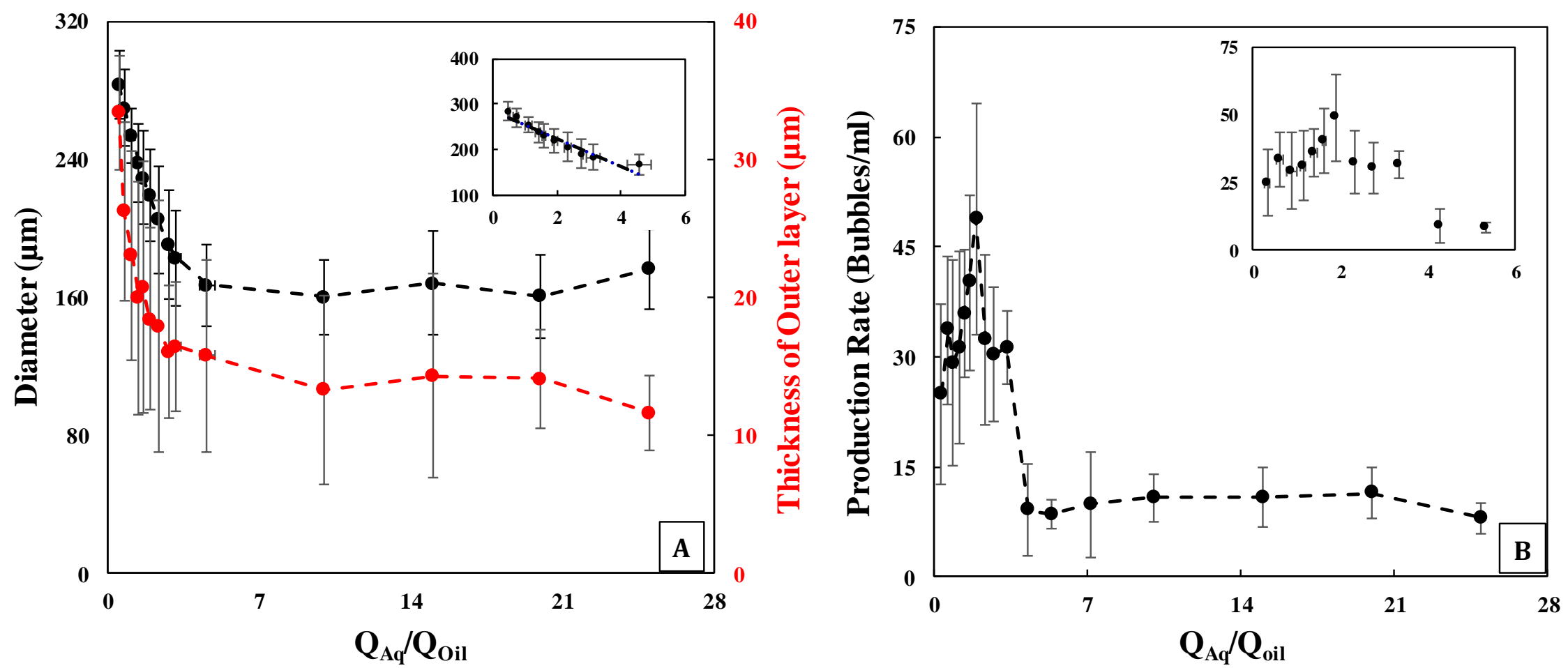

Figure 2: (A) Average size of the double layer microbubbles (black dotted line) and average oil layer thickness (red dotted line) of the double-layered microbubbles synthesized using two $\mathrm{T}-$ junctions as a function of liquid flow ratio, $\mathbf{Q}_{\mathrm{Aq}} / \mathrm{Q}_{\mathrm{oil}}$. Inset to Fig. 2 (A) presents variation in the outer diameter at lower flow ratios (B) Average production rate of the double layer microbubbles synthesized using two $\mathbf{T}$-junction as a function of liquid flow ratios. Inset to Fig 2 (B) presents variation in production rate at lower flow ratios 
At higher flow ratios $\left(\mathrm{Q}_{\mathrm{Aq}} / \mathrm{Q}_{\mathrm{oil}} \geq 5\right)$ however, the shear and the drag forces acting on the gas slug acquire a balance for a given capillary size of the T-junction resulting in no further reduction of the microbubble size. Similar observation was reported by Pancholi et al. ${ }^{40}$ for single-layered microbubbles where the authors observed that there is a limit to which an increase in flow ratios and viscosity can decrease the diameter of the bubble for a given capillary diameter and the orifice size. Fig. 2A also presents the variation in the thickness of oil layer of double-layered microbubbles (dotted red line) as a function of $\mathrm{Q}_{\mathrm{Aq}} / \mathrm{Q}_{\mathrm{oil}}$. The oil layer thickness was found to decrease as $\mathrm{Q}_{\mathrm{Aq}} / \mathrm{Q}_{\text {oil }}$ increased up to 5 and then become constant as $\mathrm{Q}_{\mathrm{Aq}} / \mathrm{Q}_{\text {oil }}$ increased beyond 5 . At lower flow ratios $(\leq 5)$, the thickness of the oil layer was higher as compared to the oil layer at higher flow ratios $(\geq 5)$. This suggests that at low $\mathrm{Q}_{\mathrm{Aq}} / \mathrm{Q}_{\mathrm{Oil}}$, the volume of oil present at the tip of the junction to encapsulate the BSA microbubble is large (due to high $\mathrm{Q}_{\text {oil }}$ ) which produces thicker double-layered microbubbles while at higher $\mathrm{Q}_{\mathrm{Aq}} / \mathrm{Q}_{\mathrm{oil}}$, the lesser oil available (due to low Qoil) at the tip of the junction produces thinner oil layered microbubbles.

Fig. 2B presents the effect of $\mathrm{Q}_{\mathrm{Aq}} / \mathrm{Q}_{\text {oil }}$ on the production rate of microbubbles. The inset presents the effect of flow ratios at low $\mathrm{Q}_{\mathrm{Aq}} / \mathrm{Q}_{\mathrm{Oil}}$. It can be observed that for $\mathrm{Q}_{\mathrm{Aq}} / \mathrm{Q}_{\mathrm{Oil}}<1$, the production rate is moderate and varies between 15 - $40 \mathrm{bubbles} / \mathrm{sec}$. The production rate increased at higher $\mathrm{Q}_{\mathrm{Aq}} / \mathrm{Q}_{\mathrm{Oil}}$ and went through a maximum, indicating that an increase in the aqueous flow rate in the second T-junction increased the number of bubbles pinched off per second in the first T-junction. However, for $\mathrm{Q}_{\mathrm{Aq}} / \mathrm{Q}_{\mathrm{oil}}$ ranging between 2 to 5 (for a constant gas pressure), the production rate decreased sharply and became constant at $\mathrm{Q}_{\mathrm{Aq}} / \mathrm{Q}_{\mathrm{Oil}}$ greater than 5. This suggests that at higher aqueous flow rates, aqueous phase in the $2^{\text {nd }} \mathrm{T}$-junction pushes the oil and gas in the first $\mathrm{T}$ junction upstream due to increased pressure and thereby resulting in a reduction of gas pinch-off frequency and hence the low bubble production rate ${ }^{41,42}$. Fig. 2B also gives an overview of range 
of bubble production rates that can be achieved for the arrangement of two T-junctions connected in series. If one operates at a highest production rate of 50 bubbles/sec corresponding to $\mathrm{Q}_{\mathrm{Aq}} / \mathrm{Q}_{\text {oil }}$ of 2,5 million bubbles $\left(5 \times 10^{6}\right)$ can be produced in approximately 3 hours. On the other hand, at a lower production rate of 10 bubbles/sec corresponding to $\mathrm{Q}_{\mathrm{Aq}} / \mathrm{Q}_{\text {oil }}$ greater than 5 , production of 5 million bubbles $\left(5 \times 10^{6}\right)$ can be achieved in approximately 14 hours.

Operating regimes for formation of double $\mathbf{T}$-junction device: During microbubble production, not always the operating conditions resulted in a successful formation of double layered microbubbles. At certain conditions, only liquid droplets were obtained or only single layered microbubbles were formed. Fig. 3 B-D presents the optical images for droplets, single layer and double layer microbubbles produced using two T-junctions in series.

In order to identify the operating conditions for the formation of double layered microbubbles, a regime map shown in Fig. 3A was generated based on the experimental observations. To generate the regime map, the capillary number for aqueous phase $\left(\mathrm{Ca}_{\mathrm{aq}}\right)$ was plotted against the capillary number for the oil phase $\left(\mathrm{Ca}_{\text {oil }}\right)$. Capillary number $(\mathrm{Ca})$ is defined as the ratio of viscous force to surface tension force, $C a=\frac{\mu v}{\sigma}, \mu$ is the solution viscosity, $\mathrm{v}$ is the liquid velocity, $\sigma$ is the surface tension. Transport properties used for calculation of capillary numbers are given in Table 1.

Table 1. Transport properties of liquid solution used in the experiments

\begin{tabular}{cccc}
\hline SN & Sample & $\begin{array}{c}\text { Viscosity } \\
(\mathbf{m P a} \times \mathbf{s e c})\end{array}$ & $\begin{array}{c}\text { Surface tension } \\
(\mathbf{m N} / \mathbf{m})\end{array}$ \\
\hline 1 & $15 \mathrm{wt} \%$ BSA, 15\% v/v Glycerol and 3.5 mM SDS & 2.7 & 52.1 \\
\hline 2 & Silicone Oil & 70 & 21.2 \\
\hline
\end{tabular}




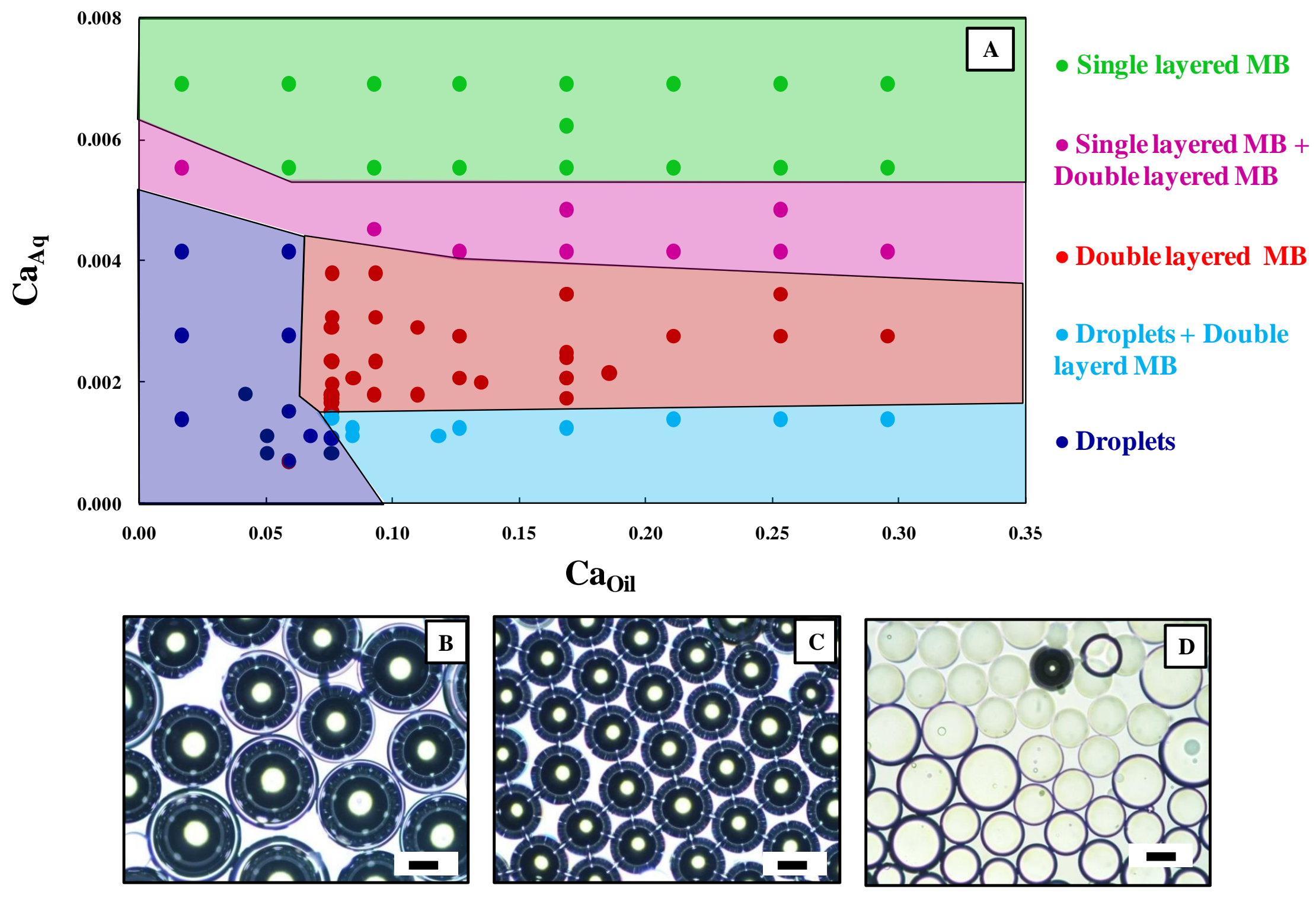


Figure 3: (A) Phase map for operation of T-junction devices connected in series. Capillary number of aqueous phase (Caaq) has been plotted against capillary number of oil phase (Cail). The gas pressure was held constant at $101.3 \mathrm{kN} / \mathrm{m}^{2}$ (gauge pressure) when the BSA solution flowrate was varied from 0.025 to $0.25 \mathrm{~mL} / \mathrm{min}$ corresponding to Caaq from 0.001 to 0.007 and the oil flowrate was varied from 0.010 to $0.175 \mathrm{~mL} / \mathrm{min}$ corresponding to Caoil from 0.017to 0.296.Violet markers represent BSA droplets, red markers represent double layered microbubbles, pink markers represent mixture of single layered and double layered microbubbles, blue marker represents mixture of droplets and double layered microbubbles and green markers represent single layered microbubbles. Optical Images of double layer microbubbles, $(B)$ double layer microbubble (DLMB), ratio of Aqueous phase $\left(\mathrm{L}_{\mathbf{1}}\right)$ to Oil phase $\left(\mathrm{L}_{2}\right)$ flowrate 1.7, (C) single layer microbubble (SLMB), $\mathbf{L}_{1} / \mathrm{L}_{2}$ ratio 3 , (D) BSA droplets $(B D L), L_{1} / L_{2}$ ratio 0.7. (Scale bar $\left.=100 \mu \mathrm{m}\right)$.

Different operating regimes observed during operation of $\mathrm{T}$-junction devices connected in series, were identified based on the extent of encapsulation of microbubbles in oil layer for a given liquid and oil flow rate and gas pressure. The $\%$ of microbubbles encapsulated at given flow rates was estimated based on Image $\mathbf{J}$ analysis of optical microscopy images of microbubble samples collected. If $\%$ encapsulation for a given flow rate condition was $\geq 80 \%$, then the operating regime was considered to fall under the double layer microbubble formation regime. If $\%$ encapsulation of double layered microbubble was $\leq 30 \%$ then it was considered to fall under the single layer microbubble formation regime. If the $\%$ encapsulation was found to vary between 30 to $75 \%$, then the regime was considered to fall under a mixed region of double layered and single layered microbubbles. When the proportions of single or double layer microbubbles were less than $10 \%$ with rest of the sample consisting of droplets, it was considered to be a droplet formation regime. 
Droplet formation regime: It can be observed from Fig. 3A that at low capillary numbers of oil phase $\left(0<\mathrm{Ca}_{\mathrm{oil}}<0.08\right)$ and low to moderate capillary numbers for aqueous phase $\left(0<\mathrm{Ca}_{\mathrm{aq}}<\right.$ 0.004), chances of liquid droplets formation were high (region highlighted as violet in a phase map shown in Fig. 3A). High-speed videos captured under these conditions (see Video S1) suggest that the majority of microbubbles emerging at the tip of the second junction were unstable. The gas slugs arriving at the tip of the junction were found to be surrounded largely by an oil phase and inadequate aqueous phase. It was observed that as the gas slug emerges out at the tip, it merges into other gas slugs which ultimately become unstable (Fig. S1 and Video S1). Under these circumstances, the aqueous phase disperses in the oil phase and forms BSA solution droplets shown in Fig. 3D.

Double layered microbubble formation regime: Upon increasing $\mathrm{Ca}_{\text {oil }}$ beyond 0.08 and $\mathrm{Ca}_{\mathrm{aq}}$ ranging between 0.002 to 0.004 , double layer microbubble formation started to occur (Fig. S2 and Video S2). Video S3 (captured for the second T-junction) and Video S4 (captured for the capillary tube of the second T-junction) show the process of double layered microbubble formation. In the first T-junction, the shear and the pressure force acting on the gas stream splits the stream into a series of small gas slugs. These gas-slugs then enter into the second $\mathrm{T}-$ junction where the aqueous phase also joins the stream of oil droplets and gas slugs (Video S3). The correct sequence of liquid and gas phases is vital for the formation of double layered microbubbles in the double $\mathrm{T}-$ junction setup. Fig. S3 presents the snapshots of second $\mathrm{T}$ junction and the outlet capillary of $\mathrm{T}$ - junction set up. It can be observed from Fig. S3A that the sequence is, gas pocket (G, Black) followed by oil (O, Transparent) followed by aqueous phase (A, Blue), [-G-O-A-]. Thus, for two consecutive gas pockets the sequence is -G-O-A-G-O-A(Fig. S3A). If the repeating units are unchanged, then the aqueous phase should come out first 
followed by oil and gas phases. Interestingly, it was observed that the sequence of phases is changed in the outlet capillary of the $\mathrm{T}$ - junction. The sequence actually changes to -G-A-O-GA-O- from -G-O-A-G-O-A- as shown in Fig. S3B. The fluid phases appear to re-arrange themselves in the capillary in the increasing order of viscosity as they move downstream where the oil phase rips through the aqueous phase resulting in a changed in sequence. Therefore, the oil phase emerges out at the tip of the T-junction setup first followed by aqueous phase and then the gas phase. As oil comes out first, the gas phase gets encapsulated in aqueous BSA layer first and then with the oil layer, resulting in a double layered microbubble.

Single layered microbubble formation regime: As the capillary number of aqueous phase $\left(\mathrm{Ca}_{\mathrm{aq}}\right)$ was increased beyond 0.004 at a given $\mathrm{Ca}_{\mathrm{oil}}$, the double layer microbubble formation continued to occur (region highlighted as red in the phase map shown in Fig. 3A) until the aqueous stream becomes dominant and disrupts the double layered microbubble formation regime. High speed videos captured at higher aqueous phase flow rate (corresponding to $\mathrm{Ca}_{\mathrm{aq}}$ > 0.005) suggest that the shear force acting on the gas pocket splits it into number of smaller bubbles (See Video S5 and S6). Out of these bubbles, only the last bubble formed out of the original gas slug can get covered in an aqueous layer as well as an oil layer and the rest of the bubbles only get covered in an aqueous layer thereby resulting formation of a small number of double-layered microbubbles and a large number of single layered microbubbles (Video S6). This mixed region consisting of single layered and double layered microbubble has been highlighted in pink in the phase map shown in Fig. 3A. The single layered microbubble formation region has been highlighted in green in the phase map shown in Fig. $3 \mathrm{~A}$. As $\mathrm{Ca}_{\mathrm{Aq}}$ is increased further, beyond 0.006, an increase in aqueous flow rate enhances the frequency of 
splitting of gas slugs into smaller bubbles resulting mostly in formation of single layered microbubbles and a very low number of double layered microbubbles.

\section{In-vitro stability of double layered microbubbles in air saturated aqueous environment:}

Fig. 4A presents dissolution kinetics of double-layered microbubbles of different initial sizes. Freshly prepared double layered microbubbles were suspended in an air-saturated aqueous environment and were imaged until the microbubbles disappeared completely. The pressure inside the microbubble is higher than the atmospheric pressure due to Laplace pressure inside the

bubble $\left(P_{g}=P_{\text {atm }}+\frac{2 \sigma}{R}\right)$. As the microbubble size reduces during dissolution, the pressure inside the microbubble increases. With an increase in the pressure inside the microbubble, the concentration gradient for diffusion of the gas from the bubble increases resulting in a further decrease in microbubble size. It was observed that as the double layered microbubble dissolved, the inner diameter of the bubble reduced in size (see Fig. 4 B-E) whereas the outer diameter of the microbubble (corresponding to the oil layer) decreased only slightly (See Video S7 in SI). As the double layered microbubble dissolved completely, it eventually transformed into an oil droplet as shown in Fig.4B (and in Video S7 in SI). It was also observed that larger microbubbles exhibited higher dissolution times as compared to the smaller bubbles which could be attributed to slower mass transfer rates in case of larger bubbles owing to lower concentration gradients for microbubble dissolution. Interestingly, no increase in microbubble size was observed during microbubble dissolution which was observed for dissolution of single layered microbubbles made of gases other than air, in aqueous air-saturated medium ${ }^{43-45}$. 


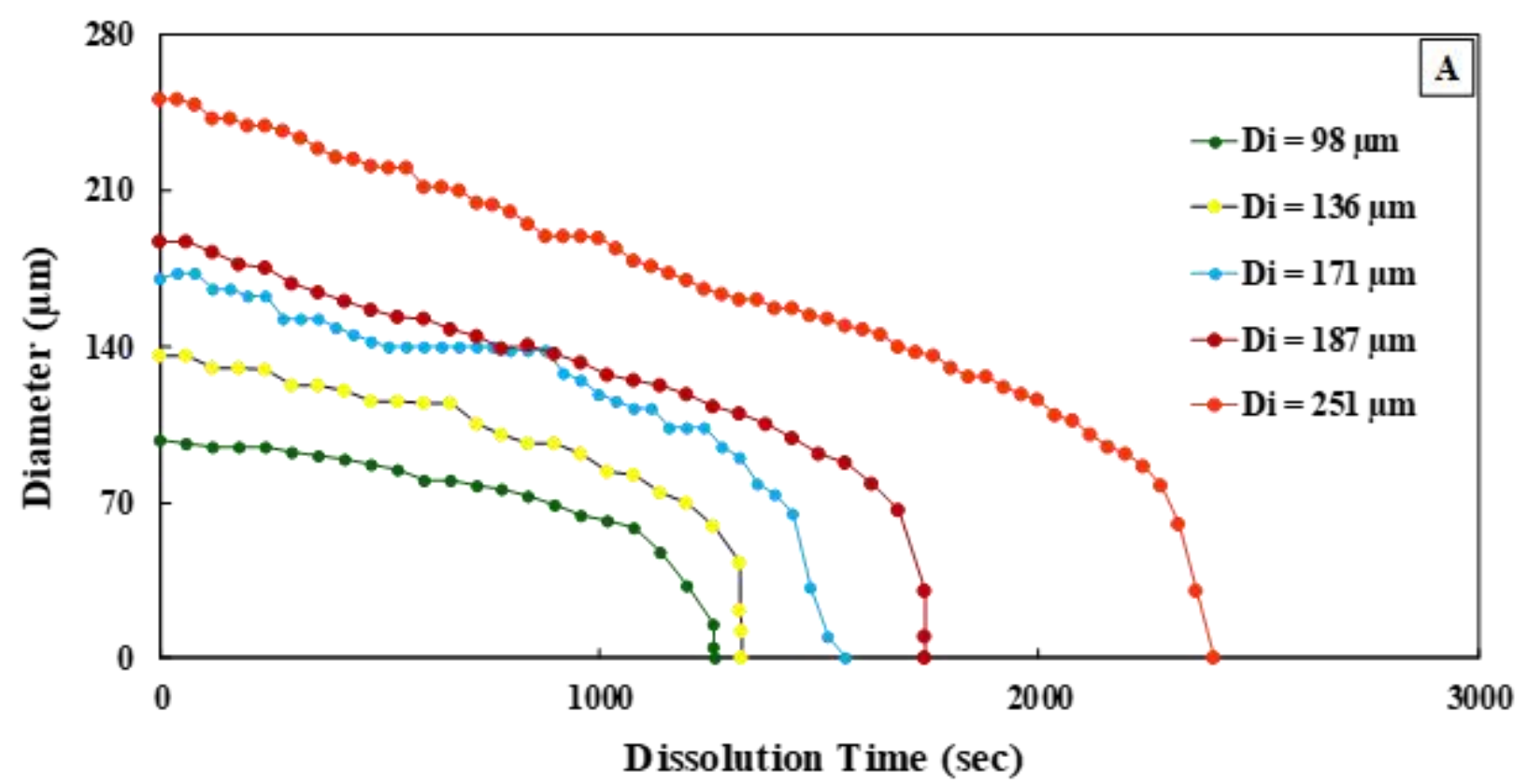

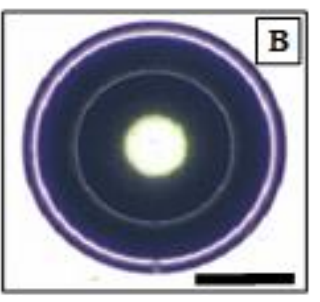

$\mathrm{t}=0 \mathrm{~min}$

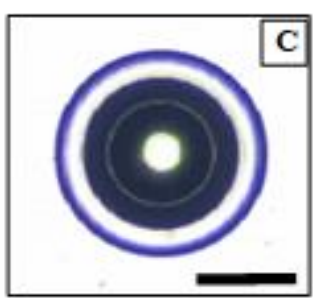

$\mathrm{t}=28 \mathrm{~min}$

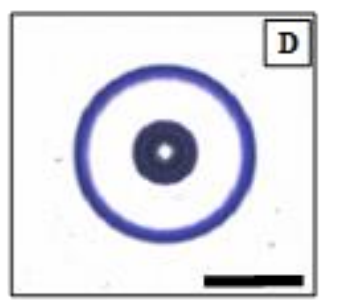

$\mathrm{t}=41 \mathrm{~min}$

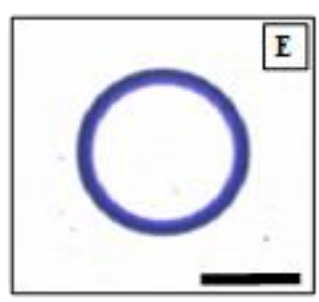

$\mathrm{t}=42 \mathrm{~min}$

Figure 4: (A) Dissolution profile of BSA-silicone oil double layered microbubbles dissolving in air saturated aqueous environment. (B-E) Optical images of different stages of double layered microbubble dissolving in an air saturated aqueous environment. $($ Scale bar $=100 \mu \mathrm{m})$ 
In-vitro Drug release from drug loaded double layered microbubbles: Fig. 5 shows the percentage (\%) drug release of curcumin from double-layered microbubbles in presence and absence of ultrasound. It can be observed that the release of curcumin from double-layered microbubbles at the end of $24 \mathrm{hrs}$ was higher in presence of ultrasound as compared to drug release in absence of ultrasound. The maximum drug release was $51.1 \pm 3.9 \%$ compared to 27.7 $\pm 1.6 \%$ drug release in the presence and absence of ultrasound respectively. This indicates that the application of ultrasound as an external trigger to the double layered microbubbles results in the higher release of curcumin from the microbubble shell. The higher release of curcumin in presence of ultrasound can be attributed to the cavitation of double-layered microbubbles where the microbubble undergoes volumetric expansion and contraction of in response to the incident ultrasound ${ }^{46}$ and due to disruption of oil layer of the double-layered microbubble in presence of ultrasound.

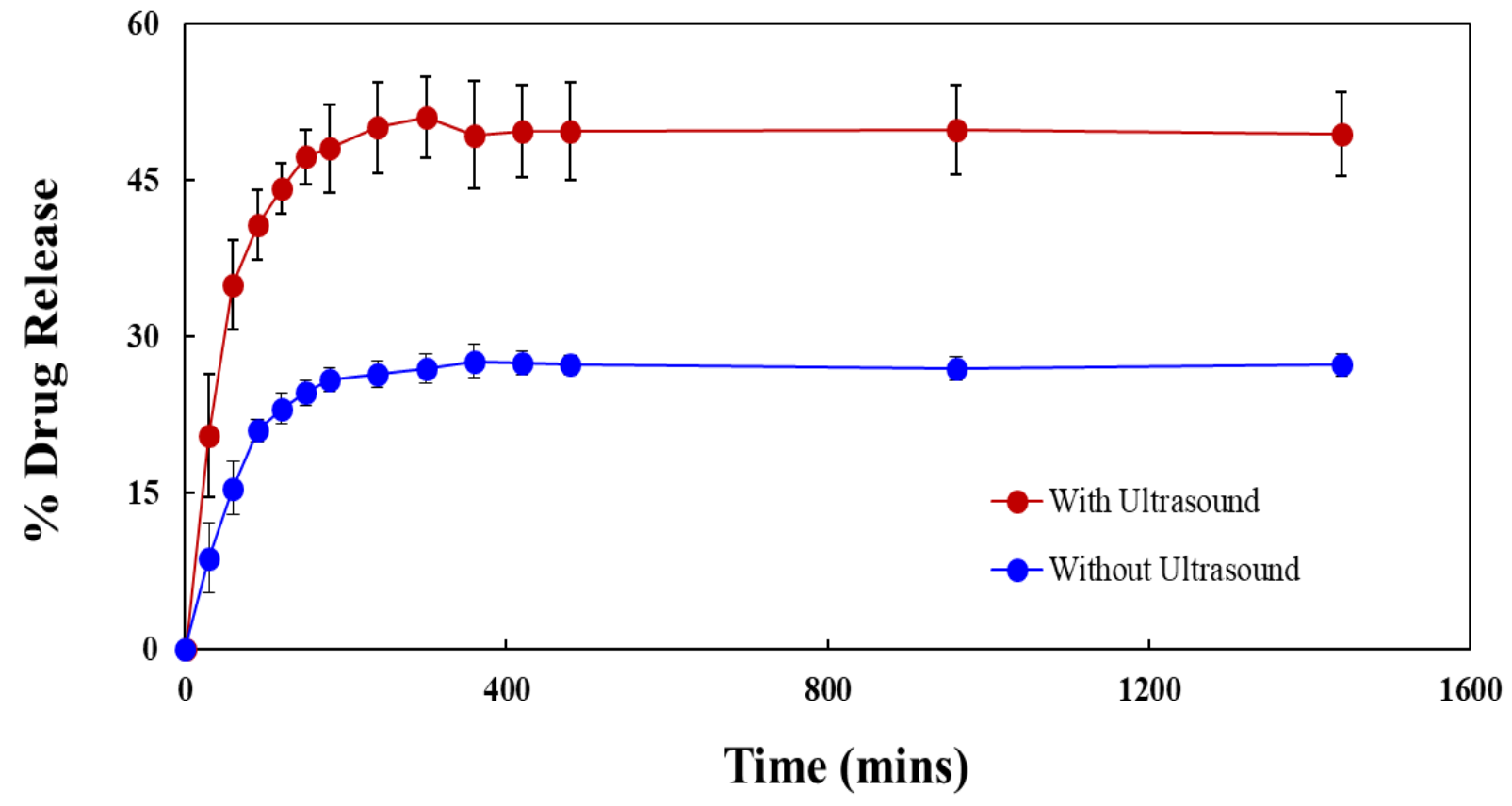

Figure 5: In-vitro release profile of curcumin from double layered microbubbles 


\section{In-vitro cytotoxicity and LIVE/DEAD assays studies for double layered microbubbles}

Fig. 6 presents MTT results obtained for MDA-MB-231 cells incubated with double layered microbubbles containing anticancer drugs such as doxorubicin and curcumin. Quantitative cell viabilities were measured with MTT assay. Cell viabilities significantly decreased with respect to control groups (A-C) in a dose-dependent manner. Doxorubicin use at 10 and $25 \mu \mathrm{M}$ concentrations exhibits moderate cytotoxicity (50-70\% cell viability) against MDA-MB-231 whereas the highest concentration of doxorubicin inhibits more than $70 \%$ of the cells. Although curcumin treatment reduced cell viabilities, curcumin was less cytotoxic at concentration below $543 \mu \mathrm{M}$, while it was moderate to highly cytotoxic at concentrations higher than $543 \mu \mathrm{M}$. However, co-delivery of two active agents creates a synergic effect and the same cytotoxicity level of $50 \mu \mathrm{M}$ doxorubicin treated sample groups was achieved with $10 \mu \mathrm{M}$ doxorubicin only with the aid of curcumin incorporation. The calculations were performed to determine the synergy between the drugs, DOX and CUR using the methodology proposed by Chou-Talalay ${ }^{47}$. Using data presented in Fig 6, the combination index (CI) for the drug combination of DOX and CUR was estimated to be 0.472 [See Supplementary Information (SI) for detailed calculations]. Since, the CI of the drug combination is less than 1, it is clear that a decrease in the cell viability of MDA-MB-231 cell lines is due to synergistic effect of DOX and CUR ${ }^{48}$ when CUR and DOX were used together in the oil and aqueous layer respectively. The interaction of double-layered microbubbles and cells is facilitated by ultrasound, mainly due to cavitation and bursting of microbubbles. Cavitation and bursting of microbubbles lead to the disruption of an oil layer in presence of ultrasound resulting in enhanced drug release in the vicinity of the cells. Moreover, the ultrasound is known to induce sonoporation in cell membranes ${ }^{49}$. The pores formed on cell 
membranes due to sonoporation in presence of ultrasound enable internalization of drug molecules released for the double-layered microbubbles leading to higher cell death.

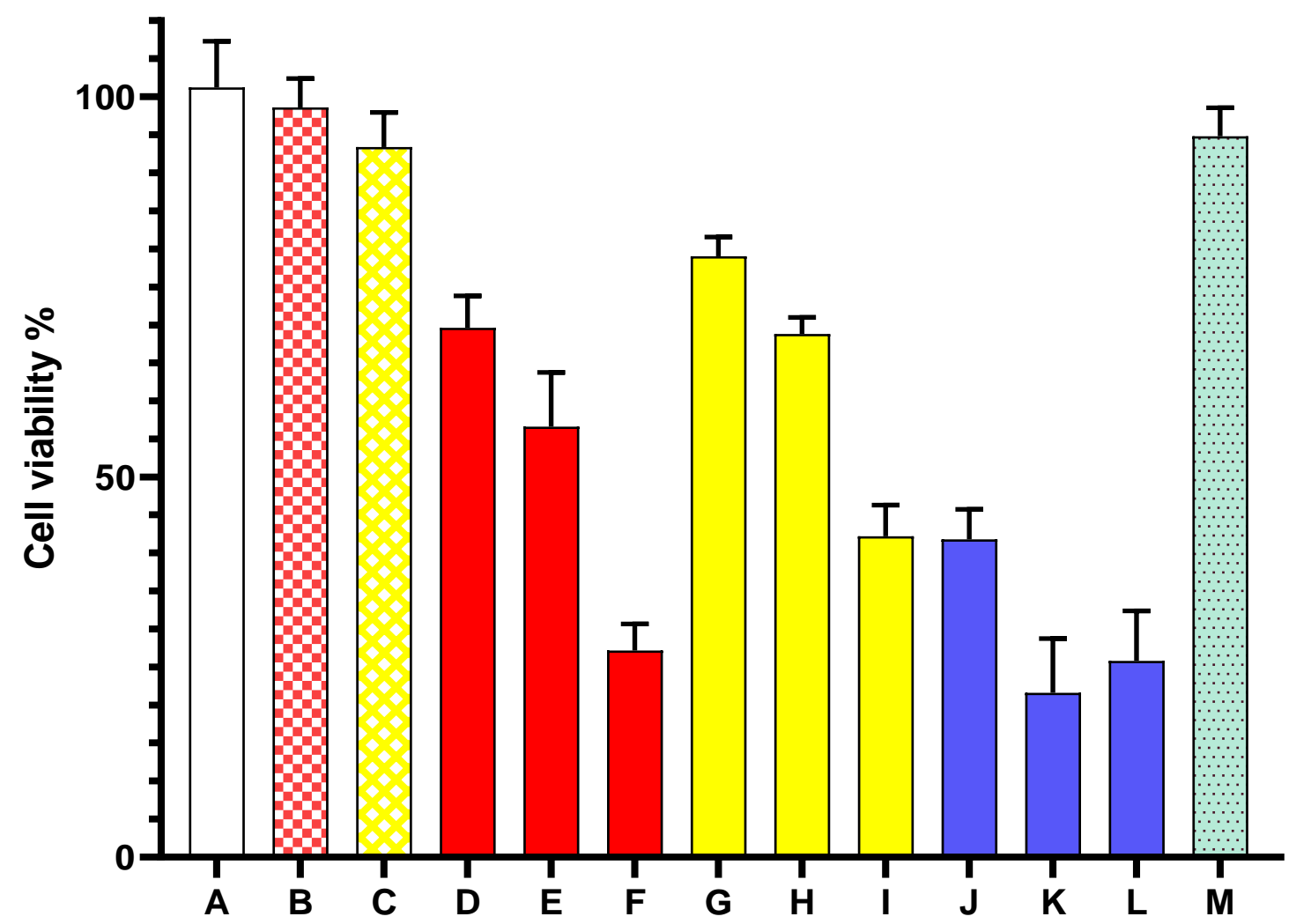

Figure 6: MTT results of MDA-MB-231 cells. MTT results of MDA-MB-231 cells. (A) control, (B) interacted with BSA microbubbles, (C) interacted with double layered microbubbles, (D-F) double layered microbubbles containing with varying concentrations of doxorubicin $(10,25$ and $50 \mu \mathrm{M}$ ) treated cells, (G-I) double layered microbubbles containing varying concentrations of $(271,542$ and $1357 \mu \mathrm{M})$ curcumin treated cells, (J-L) double layered microbubbles containing $10 \mu \mathrm{M}$ doxorubicin and varying concentrations of $(271,542$ and $1357 \mu \mathrm{M})$ curcumin treated cells, (M) interacted with ultrasound. All sample groups were interacted with microbubbles at a density of $10^{3}$ cells/well subsequent to 10 second ultrasonic treatment 
Fig. 7 shows the MDA-MB-231 cell viabilities of after interaction with microbubbles. The number of viable cells and cell morphologies shows no difference amongst control and microbubbles without active agent groups.
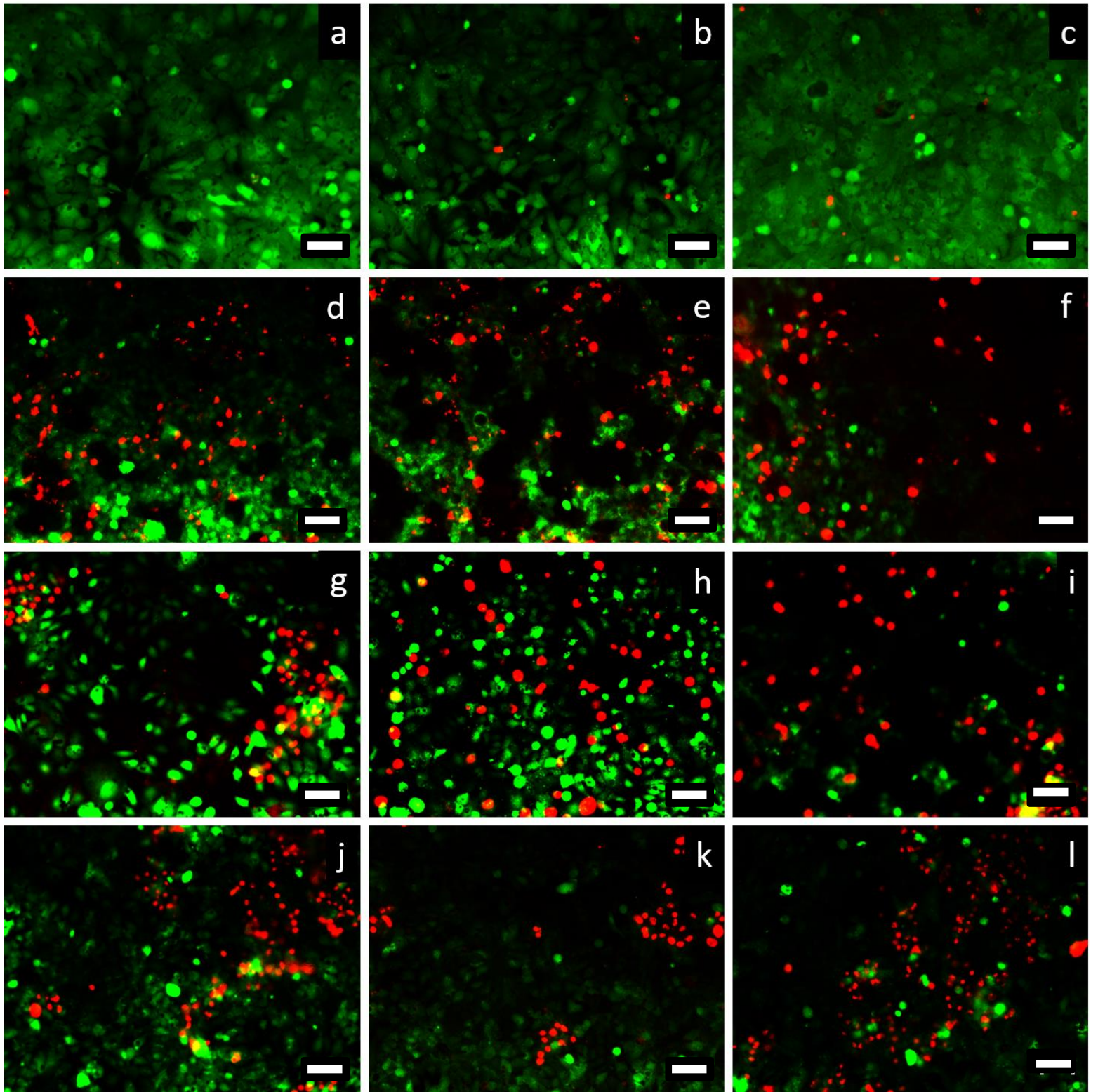

Figure 7: LIVE/DEAD stained fluorescence micrographs showing 2D culture of MDA- MB-231 cells. (a) control, (b) interacted with BSA microbubbles, (c) interacted with double layered microbubbles, (d-f) double layered microbubbles containing with varying concentrations of doxorubicin (10, 25 and $50 \mu \mathrm{M})$ treated cells, (g-i) double layered microbubbles containing with 
varying concentrations of $(271,542$ and $1357 \mu \mathrm{M})$ curcumin treated cells, $(\mathrm{j}-\mathrm{l})$ double layered microbubbles containing $10 \mu \mathrm{M}$ doxorubicin and varying concentrations of $(271,542$ and 1357 $\mu \mathrm{M})$ curcumin treated cells. Scale bar shows $100 \mu \mathrm{m}$. All sample groups were interacted with microbubbles at a density of $10^{3}$ cells/well subsequent to 10 second ultrasonic treatment.

In all drug treated sample groups number of dead (red) cells increases with the active agent concentration. Additionally, large black areas indicate cell proliferation was also inhibited. Red cells dominate the population in all doxorubicin concentrations, whereas curcumin is much more effective against cancer cells at a concentration level of $1357 \mu \mathrm{M}$. The last row (j-1) shows that curcumin presence significantly reduces the cell proliferation at low concentrations of doxorubicin indicating co-delivery of curcumin and doxorubicin can be exploited more efficiently, having the chance of eliminating side effects of doxorubicin use at high doses.

In-vitro Cell invasion of MDA-MB-231 cells: Fig. 8 presents confocal images of various 3D spheroids showing regions stained with hoechst, phalloidin and overlay of hoechst stain and phalloidin stain without and with different concentrations of curcumin treatment in presence and absence of ultrasound. The cell invasion indices calculated for the 3D tumor models investigated in this work are presented in Table 2. It is interesting to note that $3 \mathrm{D}$ tumor models treated with $20 \mu \mathrm{L}$ of CUR-MBs with ultrasound treatment exhibited a cell invasion index of 0.05 which is lower than that of the 3D tumor model treated with $20 \mu \mathrm{L}$ of CUR-MBs and also than that of the cell invasion index calculated for tumor model treated with raw CUR. It was observed that use of ultrasound results in enhanced uptake of curcumin (Fig. 8J-8L). 

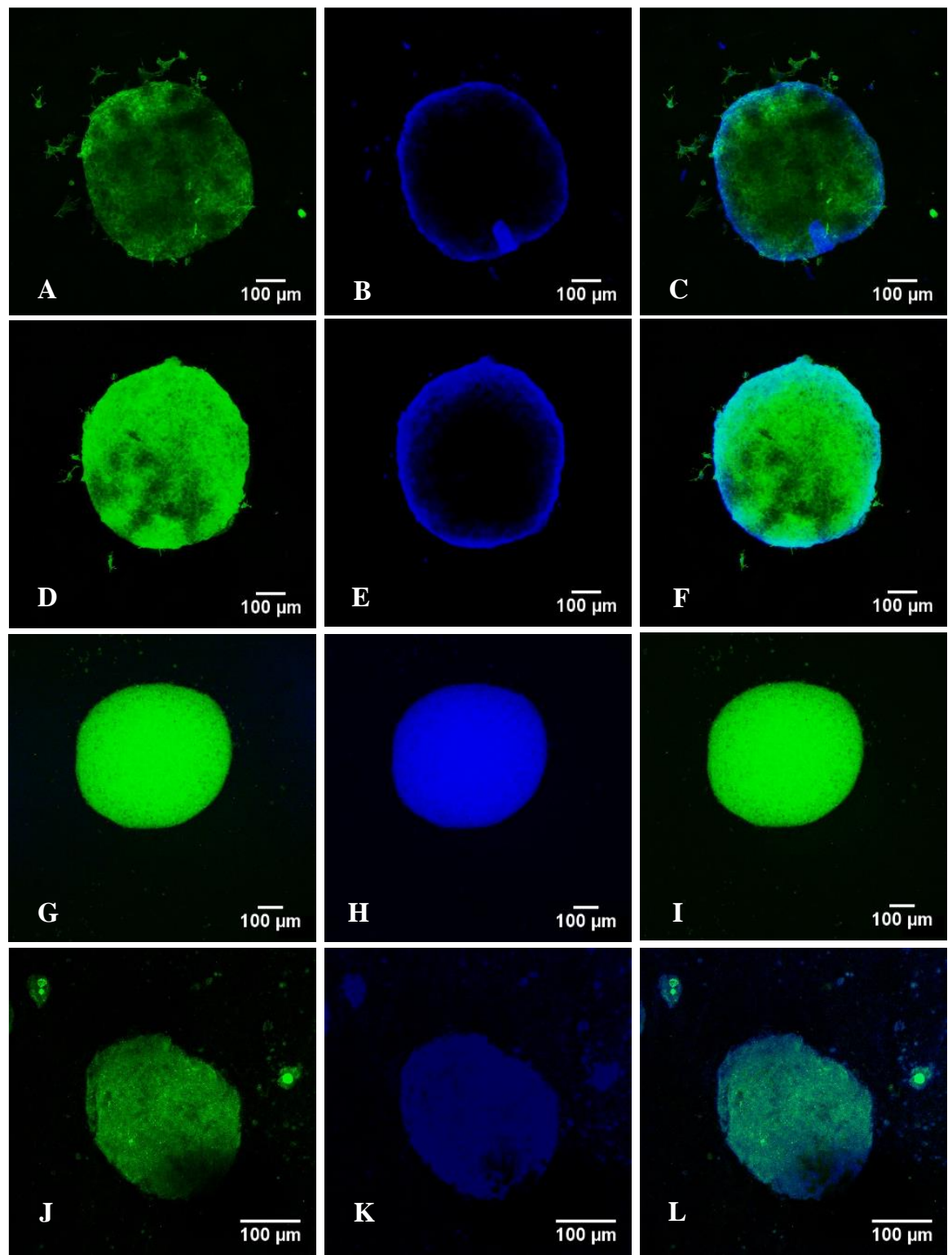

Figure 8: Confocal Images of MDA-MB-231 Breast cancer cells (A-C) untreated sample, (D-F) treated with curcumin alone, (G-I) treated with curcumin loaded double layered microbubbles without ultrasound, (J-L) treated with curcumin loaded double layered microbubbles with ultrasound. 
It is also evident that use of ultrasound influences the integrity of spheroids. The spheroid treated with CUR-MBs and ultrasound seems to be slightly disintegrated/loosened (Fig. 8J-8L) whereas the spheroid treated with CUR-MBs (and without ultrasound) remained intact (Fig. 8G-8I).

Table 2. Cell Invasion Indices calculated for double layered microbubble treated spheroids

SN

\section{Sample}

Cell Invasion Index
13 D tumour model without treatment

2 3D tumour model treated with raw CUR

3 3D tumour model treated with CUR-MBs $(20 \mu \mathrm{L})$ in absence of Ultrasound

4
3D tumour model treated with CUR-MBs $(20 \mu \mathrm{L})$ in presence of Ultrasound
0.26

0.15

0.10

0.05

Conclusions: In this work, a new method of using two micro-fluidic T-junctions in series has been described for production of double-layered microbubbles with oil as an outer layer, the aqueous protein solution as the middle layer, and nitrogen $\left(\mathrm{N}_{2}\right)$ gas as the core. The size of double layered microbubbles, thickness of oil layer and the production rate of microbubbles was found to be strongly dependent on the flow ratio of aqueous to oil phase $\left(\mathrm{Q}_{\mathrm{Aq}} / \mathrm{Q}_{\mathrm{oil}}\right)$. As $\mathrm{Q}_{\mathrm{Aq}} / \mathrm{Q}_{\text {oil }}$ increased, the size and the thickness of oil layer initially decreased linearly with the flow ratio and then became steady at higher flow ratios. The microbubble production rate however exhibited maximum with an increase in $\mathrm{Q}_{\mathrm{Aq}} / \mathrm{Q}_{\mathrm{oil}}$ initially and became steady at higher $\mathrm{Q}_{\mathrm{Aq}} / \mathrm{Q}_{\mathrm{oil}}$. The microbubble size was found to vary from $280 \pm 30 \mu \mathrm{m}$ to $100 \pm 20 \mu \mathrm{m}$ and the microbubble production rate was found to vary between $50 \pm 15$ to $10 \pm 3$ bubble/sec. The regime map was developed to identify different regimes for formation of double layered microbubbles, single 
liquid droplets and single layered microbubbles. It was observed that double layered microbubbles were formed at low to moderate capillary numbers for aqueous phase and at moderate to high capillary numbers for the oil phase. In-vitro dissolution of microbubbles was carried out in an air saturated aqueous environment where it was observed that during dissolution, the size of the microbubble only decreased and it did not show any growth phase. Nitrogen present in the core of the bubble continuously dissolved in the surrounding medium resulting in an oil droplet at the end of the dissolution process. Double layered microbubbles were loaded with varying concentration of doxorubicin $(10-50 \mu \mathrm{M})$ and curcumin $(271-1357$ $\mu \mathrm{M})$ and were delivered to MDA-MB-231 cells. It was observed that the combination of drugs produces a synergistic effect which leads higher cell deaths. The combined effect of $10 \mu \mathrm{M}$ and $543 \mu \mathrm{M}$ is equivalent to the effect of only $50 \mu \mathrm{M}$ of doxorubicin. Thus, with the combination of drugs, the harmful effects of higher doxorubicin concentration can be eliminated. Double layered microbubbles loaded with curcumin were also tested for their efficiency to inhibit cell invasion of 3D spheroids of MDA-MB-231 cell lines. It was observed that the extent of proliferation of cells was minimum for cells treated with curcumin loaded microbubbles in presence of ultrasound. This suggests that double layered microbubbles in combination with ultrasound can be used for efficient reduction of the cell proliferation from tumors.

\section{Associated content}

\section{Electronic supplementary information}

Snapshots of the tip of T-junction microfluidic device captured during droplet formation and double layered microbubble formation regimes. Snapshots of the second T-junction and the outlet capillary highlighting of the sequence of fluids. Explanation for the estimation of 
synergistic effect of Curcumin and Doxorubicin using Chou-Talalay drug combination index method. High speed video captured during droplet formation regime (Video S1). High speed videos of the second T-junction and capillary tube of the second T-junction showing the process of double layered microbubble formation (Video S2, S3, and S4). High speed videos of the second T-junction showing the process of splitting of a single gas pocket (Video S5 and S6). Video of dissolution behavior of double layered microbubble $\left(\mathrm{D}_{0}=251 \mu \mathrm{m}\right)$ dissolving in an air saturated aqueous environment (Video S7).

Acknowledgements: The authors gratefully acknowledge the funding from UGC-UKIERI Joint Research Programme (UKIERI III) through a grant no. 184-7/2018 (IC). The authors acknowledge Hacettepe University, University College London (UCL), Indian Institute of Technology Gandhinagar (IITGN) to carry out this work. The authors acknowledge the mechanical workshop facility at UCL, Central Instrumentation Facility at IITGN to carry out this work. The authors also acknowledge the financial support from Ministry of Human Resources and Development (MHRD), Government of India.

\section{References}

1. Li, H.; Yang, Y.; Zhang, M.; Yin, L.; Tu, J.; Guo, X.; Zhang, D., Acoustic characterization and enhanced ultrasound imaging of long-circulating lipid-coated microbubbles. Journal of Ultrasound in Medicine 2018, 37 (5), 1243-1256.

2. Roovers, S.; Segers, T.; Lajoinie, G.; Deprez, J.; Versluis, M.; De Smedt, S. C.; Lentacker, I., The role of ultrasound-driven microbubble dynamics in drug delivery: from microbubble fundamentals to clinical translation. Langmuir 2019, 35 (31), 10173-10191.

3. Hernot, S.; Klibanov, A. L., Microbubbles in ultrasound-triggered drug and gene delivery. Advanced drug delivery reviews 2008, 60 (10), 1153-1166.

4. Zhang, Y.; Yu, J.; Bomba, H. N.; Zhu, Y.; Gu, Z., Mechanical force-triggered drug delivery. Chemical reviews 2016, 116 (19), 12536-12563.

5. Chowdhury, S. M.; Lee, T.; Willmann, J. K., Ultrasound-guided drug delivery in cancer. Ultrasonography 2017, 36 (3), 171. 
6. Sennoga, C. A.; Kanbar, E.; Auboire, L.; Dujardin, P.-A.; Fouan, D.; Escoffre, J.-M.; Bouakaz, A., Microbubble-mediated ultrasound drug-delivery and therapeutic monitoring. Expert opinion on drug delivery 2017, 14 (9), 1031-1043.

7. Sirsi, S.; Borden, M., Microbubble Compositions, Properties and Biomedical Applications. Bubble Sci Eng Technol 2009, 1 (1-2), 3-17.

8. Kaya, M.; Feingold, S.; Hettiarachchi, K.; Lee, A. P.; Dayton, P. A., Acoustic responses of monodisperse lipid encapsulated microbubble contrast agents produced by flow focusing. Bubble Science, Engineering \& Technology 2010, 2 (2), 33-40.

9. Upadhyay, A.; Dalvi, S. V., Synthesis, characterization and stability of BSA-encapsulated microbubbles. RSC Advances 2016, 6 (18), 15016-15026.

10. Upadhyay, A.; Dalvi, S. V., Microbubble formulations: synthesis, stability, modeling and biomedical applications. Ultrasound in medicine \& biology 2018.

11. Nakata, M.; Tanimura, N.; Koyama, D.; Krafft, M. P., Adsorption and Desorption of a Phospholipid from Single Microbubbles under Pulsed Ultrasound Irradiation for Ultrasound-Triggered Drug Delivery. Langmuir 2019, 35 (31), 10007-10013.

12. Tan, J.-K. Y.; Pham, B.; Zong, Y.; Perez, C.; Maris, D. O.; Hemphill, A.; Miao, C. H.; Matula, T. J.; Mourad, P. D.; Wei, H., Microbubbles and ultrasound increase intraventricular polyplex gene transfer to the brain. Journal of Controlled Release 2016, 231, 86-93.

13. Kopechek, J. A.; Carson, A. R.; McTiernan, C. F.; Chen, X.; Hasjim, B.; Lavery, L.; Sen, M.; Grandis, J. R.; Villanueva, F. S., Ultrasound targeted microbubble destruction-mediated delivery of a transcription factor decoy inhibits STAT3 signaling and tumor growth. Theranostics 2015, 5 (12), 1378.

14. Aramide, B.; Kothandaraman, A.; Edirisinghe, M.; Jayasinghe, S. N.; Ventikos, Y., General Computational Methodology for Modeling Electrohydrodynamic Flows: Prediction and Optimization Capability for the Generation of Bubbles and Fibers. Langmuir 2019, 35 (31), 10203-10212.

15. Chen, Z.; Pulsipher, K. W.; Chattaraj, R.; Hammer, D. A.; Sehgal, C. M.; Lee, D., Engineering the Echogenic Properties of Microfluidic Microbubbles Using Mixtures of Recombinant Protein and Amphiphilic Copolymers. Langmuir 2019, 35 (31), 10079-10086.

16. Gormley, C. A.; Keenan, B. J.; Buczek-Thomas, J. A.; Pessoa, A. C. S. N.; Xu, J.; Monti, F.; Tabeling, P.; Holt, R. G.; Nagy, J. O.; Wong, J. Y., Fibrin-Targeted Polymerized Shell Microbubbles as Potential Theranostic Agents for Surgical Adhesions. Langmuir 2019, 35 (31), 10061-10067.

17. Juang, E. K.; De Cock, I.; Keravnou, C.; Gallagher, M. K.; Keller, S. B.; Zheng, Y.; Averkiou, M., Engineered 3D Microvascular Networks for the Study of Ultrasound-Microbubble-Mediated Drug Delivery. Langmuir 2019, 35 (31), 10128-10138.

18. Kothandaraman, A.; Alfadhl, Y.; Qureshi, M.; Edirisinghe, M.; Ventikos, Y., Effect of the Mixing Region Geometry and Collector Distance on Microbubble Formation in a Microfluidic Device Coupled with ac-dc Electric Fields. Langmuir 2019, 35 (31), 10052-10060.

19. Zhang, Y.; Wang, L., Experimental investigation of bubble formation in a microfluidic T-shaped junction. Nanoscale and Microscale Thermophysical Engineering 2009, 13 (4), 228-242.

20. Garstecki, P.; Fuerstman, M. J.; Stone, H. A.; Whitesides, G. M., Formation of droplets and bubbles in a microfluidic T-junction-scaling and mechanism of break-up. Lab on a Chip 2006, 6 (3), 437446.

21. Garbin, V., Dynamics of Coated Microbubbles in Ultrasound. In The Micro-World Observed by Ultra High-Speed Cameras, Springer: 2018; pp 357-374.

22. Jiang, X.; Zhang, Y.; Edirisinghe, M.; Parhizkar, M., Combining microfluidic devices with coarse capillaries to reduce the size of monodisperse microbubbles. RSC advances 2016, 6 (68), 63568-63577.

23. Xiong, R.; Bai, M.; Chung, J. N., Formation of bubbles in a simple co-flowing micro-channel. Journal of Micromechanics and Microengineering 2007, 17 (5), 1002. 
24. Castro-Hernández, E.; Van Hoeve, W.; Lohse, D.; Gordillo, J. M., Microbubble generation in a co-flow device operated in a new regime. Lab on a Chip 2011, 11 (12), 2023-2029.

25. Fu, T.; Ma, Y.; Funfschilling, D.; Li, H. Z., Bubble formation and breakup mechanism in a microfluidic flow-focusing device. Chemical Engineering Science 2009, 64 (10), 2392-2400.

26. Nie, Z.; Seo, M.; Xu, S.; Lewis, P. C.; Mok, M.; Kumacheva, E.; Whitesides, G. M.; Garstecki, P.; Stone, H. A., Emulsification in a microfluidic flow-focusing device: effect of the viscosities of the liquids. Microfluidics and Nanofluidics 2008, 5 (5), 585-594.

27. Bayram, C.; Jiang, X.; Gultekinoglu, M.; Ozturk, S.; Ulubayram, K.; Edirisinghe, M., Biofabrication of Gelatin Tissue Scaffolds with Uniform Pore Size via Microbubble Assembly. Macromolecular Materials and Engineering 2019, 304 (11), 1900394.

28. Gultekinoglu, M.; Jiang, X.; Bayram, C.; Wu, H.; Ulubayram, K.; Edirisinghe, M., Self-assembled micro-stripe patterning of sessile polymeric nanofluid droplets. Journal of colloid and interface science 2020, 561, 470-480.

29. Arakawa, T.; Yamamoto, T.; Shoji, S., Micro-bubble formation with organic membrane in a multiphase microfluidic system. Sensors and Actuators A: Physical 2008, 143 (1), 58-63.

30. Chen, R.; Dong, P.-F.; Xu, J.-H.; Wang, Y.-D.; Luo, G.-S., Controllable microfluidic production of gas-in-oil-in-water emulsions for hollow microspheres with thin polymer shells. Lab on a Chip 2012, 12 (20), 3858-3860.

31. Sang, F. N.; Chen, Z.; Wang, Y. D.; Xu, J. H., Dynamic formation and scaling law of hollow droplet with gas/oil/water system in dual-coaxial microfluidic devices. AlChE Journal 2018, 64 (2), 730-739.

32. Shih, R.; Bardin, D.; Martz, T. D.; Sheeran, P. S.; Dayton, P. A.; Lee, A. P., Flow-focusing regimes for accelerated production of monodisperse drug-loadable microbubbles toward clinical-scale applications. Lab on a Chip 2013, 13 (24), 4816-4826.

33. Wan, J.; Stone, H. A., Microfluidic generation of a high volume fraction of bubbles in droplets. Soft Matter 2010, 6 (19), 4677-4680.

34. Wang, W.-T.; Chen, R.; Xu, J.-H.; Wang, Y.-D.; Luo, G.-S., One-step microfluidic approach for controllable production of gas-in-water-in-oil (G/W/O) double emulsions and hollow hydrogel microspheres. Rsc Advances 2014, 4 (32), 16444-16448.

35. Churchman, A. H.; Mico, V.; De Pablo, J. G.; Peyman, S. A.; Freear, S.; Evans, S. D., Combined flow-focus and self-assembly routes for the formation of lipid stabilized oil-shelled microbubbles. Microsystems \& Nanoengineering 2018, 4 (1), 1-8.

36. Poincloux, R.; Collin, O.; Lizárraga, F.; Romao, M.; Debray, M.; Piel, M.; Chavrier, P., Contractility of the cell rear drives invasion of breast tumor cells in 3D Matrigel. Proceedings of the National Academy of Sciences 2011, 108 (5), 1943-1948.

37. Romano, M. R.; Ferrara, M.; Gatto, C.; Giurgola, L.; Zanoni, M.; Angi, M.; Rinaldi, M.; Borgia, A.; Sorrentino, T.; Tóthová, J. D. A., Safety of silicone oils as intraocular medical device: An in vitro cytotoxicity study. Experimental Eye Research 2020, 108018.

38. Mojsiewicz-Pieńkowska, K.; Jamrógiewicz, M.; Szymkowska, K.; Krenczkowska, D., Direct human contact with siloxanes (silicones)-safety or risk part 1. Characteristics of siloxanes (silicones). Frontiers in pharmacology 2016, 7, 132.

39. Attieh, Y.; Clark, A. G.; Grass, C.; Richon, S.; Pocard, M.; Mariani, P.; Elkhatib, N.; Betz, T.; Gurchenkov, B.; Vignjevic, D. M., Cancer-associated fibroblasts lead tumor invasion through integrin- $\beta 3-$ dependent fibronectin assembly. Journal of Cell Biology 2017, 216 (11), 3509-3520.

40. Pancholi, K.; Stride, E.; Edirisinghe, M., Dynamics of bubble formation in highly viscous liquids. Langmuir 2008, 24 (8), 4388-4393.

41. Parhizkar, M.; Edirisinghe, M.; Stride, E., Effect of operating conditions and liquid physical properties on the size of monodisperse microbubbles produced in a capillary embedded T-junction device. Microfluidics and nanofluidics 2013, 14 (5), 797-808. 
42. Parhizkar, M.; Edirisinghe, M.; Stride, E., The effect of surfactant type and concentration on the size and stability of microbubbles produced in a capillary embedded T-junction device. Rsc Advances 2015, 5 (14), 10751-10762.

43. Khan, A.; Dalvi, S., Kinetics of Albumin Microbubble Dissolution in Aqueous Medium. Soft Matter 2020, 16, 2149-2163.

44. Kwan, J. J.; Borden, M. A., Microbubble dissolution in a multigas environment. Langmuir 2010, $26(9), 6542-6548$.

45. Dalvi, S. V.; Joshi, J. R., Modeling of microbubble dissolution in aqueous medium. Journal of colloid and interface science 2015, 437, 259-269.

46. Sirsi, S. R.; Borden, M. A., State-of-the-art materials for ultrasound-triggered drug delivery. Advanced drug delivery reviews 2014, 72, 3-14.

47. Elwakeel, A.; Soudan, H.; Eldoksh, A.; Shalaby, M.; Eldemellawy, M.; Ghareeb, D.; Abouseif, M.; Fayad, A.; Hassan, M.; Saeed, H., Implementation of the Chou-Talalay method for studying the in vitro pharmacodynamic interactions of binary and ternary drug combinations on MDA-MB-231 triple negative breast cancer cells. Synergy 2019, 8, 100047.

48. Chou, T.-C., Drug combination studies and their synergy quantification using the Chou-Talalay method. Cancer research 2010, 70 (2), 440-446.

49. Grainger, S. J.; Serna, J. V.; Sunny, S.; Zhou, Y.; Deng, C. X.; El-Sayed, M. E., Pulsed ultrasound enhances nanoparticle penetration into breast cancer spheroids. Molecular pharmaceutics 2010, 7 (6), 2006-2019.

\section{Table of Contents Graphic Only}
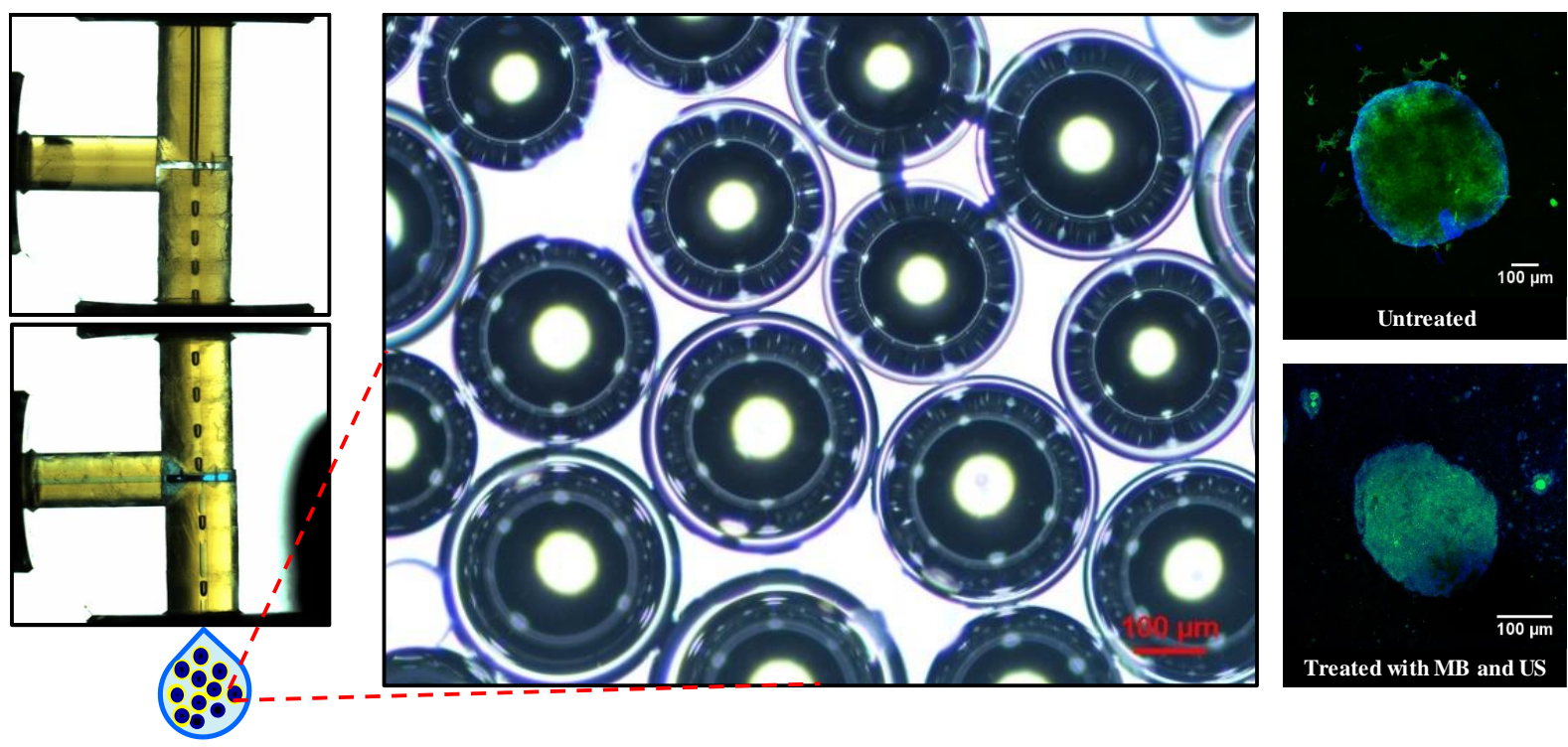